\title{
James Webb Space Telescope
}

\section{Hervey S. Stockman}

Space Telescope Science Institute, 3700 San Martin Drive, Baltimore, MD 21218, USA

email: stockman@stsci.edu

\begin{abstract}
The James Webb Space Telescope (JWST) is the scientific successor to the Hubble and Spitzer missions. Its wavelength range $(1-28 \mu \mathrm{m})$ and sensitivity $(1 \mathrm{nJy}-1 \mu \mathrm{Jy})$ complement the submillimeter facilities of the coming decade, Herschel and ALMA. The JWST development is on schedule for a June 2013 launch to L2 on an Ariane 5.
\end{abstract}

Keywords. space vehicles, space vehicles: instruments, telescopes

\section{Introduction}

In 2000, the Astronomy and Astrophysics Survey Committee of the US National Academy of Sciences recommended the James Webb Space Telescope (JWST, Webb) as the highest priority new space facility for the decade and a Giant Segmented Mirror Telescope (GSMT) as the highest priority ground based facility. These ambitious projects are the scientific successors to the very successful Hubble Space Telescope and the 8 - $10 \mathrm{~m}$ ground-based telescopes developed in the 1990s. While the emphasis in both new initiatives is for superb imaging and sensitivity in the near infrared $(1-5 \mu \mathrm{m})$, the equally exciting prospect for $W e b b$ was to extend the 'discovery space' of the Spitzer mission in the mid-infrared $(5-28 \mu \mathrm{m})$. The 'discovery space' improvement that $W e b b$ offers over GSMT and Spitzer is shown in Fig. 1.

The superb sensitivity of Webb at long wavelengths is shown in Fig. 2. along with similar estimates for the Herschel mission to be launched in 2008 and the Atacoma Large Milllimeter Array (ALMA). Also shown are sample spectra of two sources with spectra that peak in the $60-200 \mu \mathrm{m}$ : a low-mass Class 0 protostar and a high-redshift luminous infrared galaxy (starburst or AGN). This figure shows the complementarity of the three facilities in the mid-IR and sub-millimeter range.

\section{Science goals}

The Webb science goals are described in The James Webb Space Telescope (Gardner et al. 2006). They fall into four major areas listed below and are illustrative of the scientific power of the Webb. Recently, the discovery of dozens of eclipsing exo-planets by photometric surveys of stars both near the Sun and toward the Galactic Center has raised the potential of Webb studying the atmospheric properties of planets, down to Earth-like masses for nearby systems.

- First Light: The first luminous objects and the epoch of re-ionization

- Galaxy Assembly: the origins and growth of galactic structures and cosmo-chemistry

- The Birthplaces of Stars: The environments of star and planet formation

- Planets and Life: The evolution of planetary systems and the ways they could support life

\section{Project status}

The $W e b b$ development is a collaborative effort involving NASA, the European Space Agency and the Canadian Space Agency. Goddard Space Flight Center (NASA) is the 


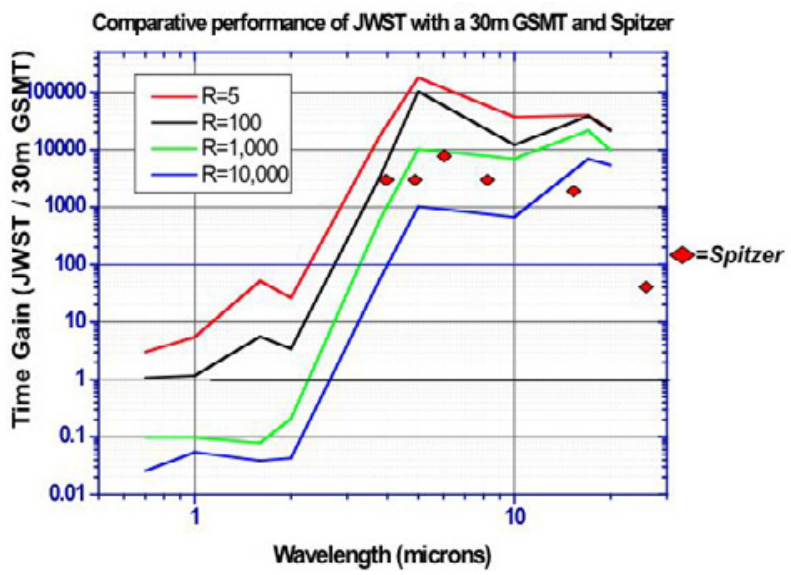

Figure 1. 'Webb discovery space compared to GSMT and Spitzer

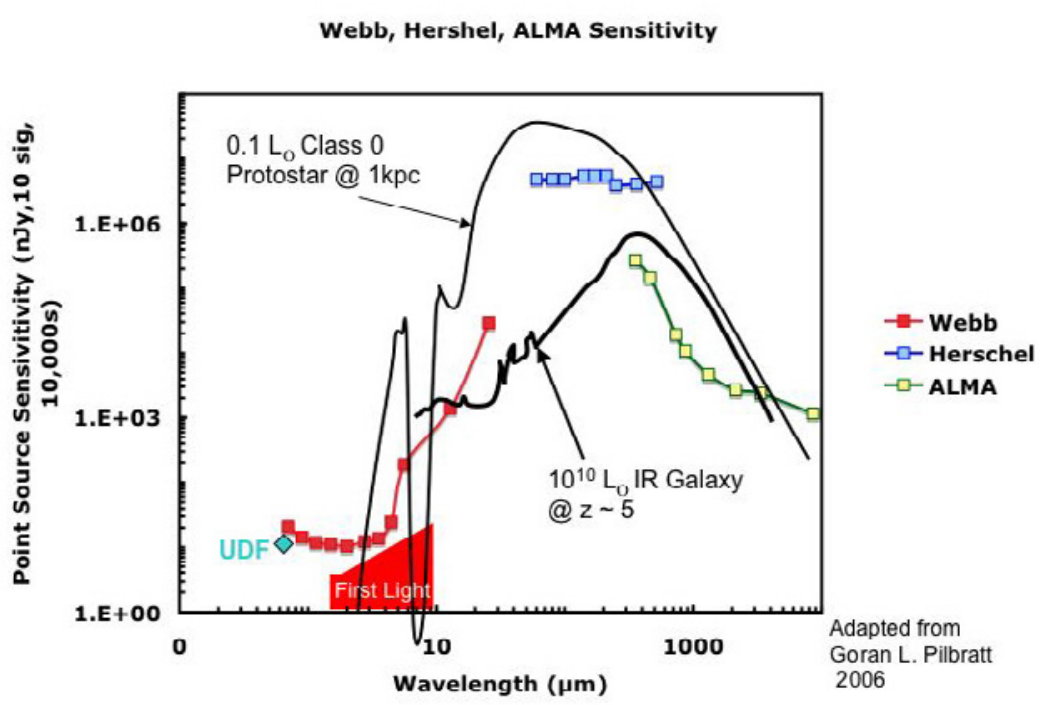

Figure 2. Webb, Herschel, and ALMA sensitivities in the mid-IR and sub-millimeter region. $10 \sigma, 10^{4} s$ observations.

lead organization and Northrop Grumman Space Technologies (NGST) is the prime contractor. The Project is on schedule for a June 2013 launch and will have demonstrated the flight worthiness of the key enabling technologies by January 2007. For a list of the SWG members and current information, see <www.jwst.gsfc.nasa.gov> and <www.stsci.edu/jwst>.

\section{References}

Gardner, J. P., Mather, J. C., Clampin, M., Doyon, R., Greenhouse, M. A., Hammel, H. B., Hutchings, J. B., Jakobsen, P., Lilly, S. J., Long, K. S., Lunine, J. I., McCaughrean, M. J., Mountain, M., Nella, J., Rieke, G. H., Rieke, M. J., Rix, H.-W., Smith, E. P., Sonneborn, G., Staivelli, M., Stockman, H. S., Windhorst, R. A., Wright, G. S., 2006, Space Sci. Rev., 123,485 